\title{
eNotfallmedizin: Notfallversorgung der Zukunft?
}

\begin{abstract}
Thomas C. Sauter ${ }^{a}$, Wolf E. Hautz ${ }^{b}$, Beat Lehmann ${ }^{c}$, Aristomenis K. Exadaktylos ${ }^{d}$
a Oberarzt, Leiter Bildung, eHealth und Telenotfallmedizin, Universitäres Notfallzentrum, Inselspital, Universitätsspital Bern, und Lernzentrum der Charité Universitätsmedizin Berlin; ${ }^{b}$ Leitender Arzt, Leiter Forschung, Universitäres Notfallzentrum, Inselspital, Universitätsspital Bern; ${ }^{\mathrm{c}}$ Stv. Chefarzt, Universitäres Notfallzentrum, Inselspital, Universitätsspital Bern; ${ }^{d}$ Chefarzt und Klinikdirektor, Universitäres Notfallzentrum, Inselspital, Universitätsspital Bern, und Co-Präsident der Schweizer Gesellschaft für Notfall- und Rettungsmedizin SGNOR
\end{abstract}

Die Schweiz hinkt der Digitalisierung der Medizin hinterher. Das Potential, welches die künstliche Intelligenz, aber auch die Telemedizin bieten, wird nur zögerlich genutzt. Und dies, obwohl sich der Wunsch seitens der Patienten nach einer niederschwelligen und zeitnahen Versorgung sowohl im ambulanten Bereich als auch bei den Notfallkonsultationen in Spitälern widerspiegelt. Um diesem Missstand entgegenwirken zu können, richtet die Universität Bern am Universitären Notfallzentrum des Inselspitals eine Assistenzprofessur für Telenotfallmedizin ein - mit dem Ziel, Ärztinnen und Ärzte in diesem Bereich auszubilden und Forschung zu betreiben.

Grundversorger sind mit einer zunehmenden Anzahl an Notfallpatienten und entsprechend anstrengenden Notfalldiensten konfrontiert, was die Bereitschaft von jüngeren Hausärzten, Notfalldienste zu leisten, reduziert [1]. Die Kombination aus einer grossen Zahl von Hausärzten, die in den nächsten Jahren in Pension gehen, zusammen mit dem Bedürfnis von jungen Ärzten nach geregelten Arbeitszeiten führt zu einem vor allem in den ländlichen Gebieten spürbaren Hausärztemangel und zu zunehmenden Schwierigkeiten, Notfalldienste adäquat abzudecken [2]. Die Bereitschaft,

\section{Ärztinnen und Ärzte sind insbesondere in Notfalldiensten zunehmend mit Patienten konfrontiert, die durch das Internet vorab informiert und oft auch verunsichert sind.}

als Arzt seine Wochenenden und Feiertage im Notfalldienst zu verbringen, nimmt ab, und eine Diskussion über den hausärztlichen Notfalldienst der Zukunft ist lanciert $[1,3]$.

Wie auch im ambulanten Bereich nimmt die Zahl der Notfallkonsultationen in an Krankenhäuser angeschlossenen Notfallstationen stetig zu [4].

Zusätzlich zur steigenden Anzahl an Patientenkontakten ist der Wunsch der Patienten nach einer zeitnahen Versorgung unabhängig von Tageszeit oder Wochentagen spürbar [3].
Zudem sind Ärzte insbesondere in Notfalldiensten zunehmend mit Patienten konfrontiert, die durch das Internet vorab informiert oder oft auch verunsichert sind. Bis jetzt unbekannte, komplexe und für viele Ärzte neuartige Fragestellungen müssen kompetent gemeistert werden, wenn Smart Devices Patienten mit einem «von einer Uhr diagnostizierten Vorhofflimmern» zu Notfallkonsultationen schicken. Gleichzeitig ist eine steigende Zahl von Patienten in einem Telemedizin-Modell versichert und kommt mit telemedizinischen Vorabklärungen und dadurch induzierten Erwartungen zu einem Notfallarzt.

Obwohl in einer grossen Erhebung der Bertelsmann Stiftung zum Stand der Digitalisierung die Schweiz nur auf Platz 14 in Bezug auf Durchdringung der Digitalisierung in den Alltag ist [5], besteht ein grosses Potential in der digitalen Transformation der Medizin in der Schweiz, welche durch die nationale Strategie «eHealth Schweiz 2.0» (2018) des Bundes und eHealth Suisse als nationale Koordinierungsbehörde unterstützt wird. Im Swiss eHealth Barometer 2019 wird die Digitalisierung von allen Gesundheitsfachpersonen als positiv wahrgenommen, aber vor allem die Vernetzung kommt nur zögernd voran. Im Swiss Primary Care Active Monitoring wird schon die Nutzung von grundlegenden Angeboten, wie elektronischen Patientendossiers, durch nur eine Minderheit der Praxen bemängelt und die Arbeit als Einzelkämpfer ohne 
Nutzung der auch digital möglichen Vernetzung und Zusammenarbeit über räumliche Gegebenheiten hinweg als vergebene Chance aufgezeigt [2].

\section{eNotfallmedizin: die Herausforderungen und Chancen}

Eine telemedizinische, videounterstützte Beratung in Echtzeit wird aktuell von weniger als 10\% der Ärzte in der Schweiz angeboten. Die Nutzung zeitversetzter Technologien wie z.B. der telemedizinischen Beurteilung von Bildern oder Befunden kommt unverändert seit 2014 nur bei einem Fünftel der Ärzte zur Anwendung [6]. Auch die Möglichkeit, Rezepte direkt auf elektronischem Weg den Apotheken zuzusenden, hat sich noch nicht in der Breite durchgesetzt [6].

\section{Auch im Sinne einer digitalen Netzwerk- bildung könnten diese digitalen Hilfsmittel viele Prozesse vereinfachen.}

All diese Aspekte könnten nicht nur als Angebot gegenüber dem Patienten den Komfort und die Zufriedenheit mit der Notfallversorgung verbessern, sondern auch ein Wettbewerbsvorteil für Ärzte sein.

Auch im Sinne einer digitalen Netzwerkbildung der Ärzte untereinander könnten diese digitalen Hilfsmittel viele Prozesse vereinfachen. Ein solches digitales Netzwerk könnte das «Einzelkämpferdasein» als oft genanntes Hindernis für die Aufnahme einer Hausarzttätigkeit in ländlichen Gebieten abmildern.

Kleinere Spitäler kommen zunehmend an ihre Grenzen, den Spagat meistern zu müssen zwischen einer Notfallversorgung rund um die Uhr auf fachärztlichem Niveau und dem steigenden Kostendruck und Mangel an Ärzten, die bereit sind, im Schichtdienst zu arbeiten. Auch hier könnte eine Netzwerkbildung mit einem Zentrumsspital eine wertvolle Hilfe sein. Im Speziellen können Telekonsilien den niederschwelligen Zugang zu Spezialisten ermöglichen und Wartezeiten für Patienten oder unnötige Verlegungen vermeiden. Dies geht weit über die lange praktizierte Möglichkeit der radiologischen telemedizinischen Befundung hinaus.

Der Einsatz von Systemen für den direkten Patientengebrauch, sogenannte Symptom-Checker, mit welchen Patienten eine Selbsttriage oder Selbstdiagnose durchführen können, nimmt rapide zu und wird zur gesellschaftlichen Realität [7]. Ein solcher Symptom-Checker meldet inzwischen mehr als 15 Millionen monatliche Besuche, und mehr als ein Drittel der US-Erwachsenen versucht eine Selbstdiagnose mit digitalen Hilfsmitteln [7]. Apps, die vollständig die Diagnosestellung
z.B. mit eigenen Fotos von der Haut ermöglichen, sind ebenfalls verfügbar [8].

Im Gegensatz zu dieser zunehmenden Verbreitung ist die Genauigkeit und Funktionalität dieser Systeme sehr unterschiedlich. Semigran et al. massen die Genauigkeit der Selbstdiagnose und der Triage-Ratschläge von 23 Symptom-Checkern mit einer grossen Zahl von Fehldiagnosen [9]. Die richtige Diagnose wurde nur in circa einem Drittel der standardisierten Patientenbewertungen an erster Stelle aufgeführt. Kontrollierte Studien, die die Sicherheit und Wirksamkeit solcher Programme nachweisen könnten, sind dringend nötig [10, 11].

Neue Techniken mit Fähigkeiten, die nicht ohne weiteres verständlich sind, wie z.B. ein auf Maschinenlernen basierendes Programm, das bei Patienten mit intermittierendem Vorhofflimmern im SinusrhythmusIntervall-EKG die Prädisposition zum Vorhofflimmern erkennen kann [12], zeigen die Notwendigkeit, sich mit diesen Techniken auseinanderzusetzen. Die Einordnung und Anwendung vergleichbarer Anwendungen wird in Zukunft eine fachliche Expertise und wissenschaftliche Evaluation benötigen, hat aber das Potential, die Notfallversorgung grundlegend zu verändern. Im täglichen Leben und Arbeiten hat die Nutzung digitaler Hilfsmittel von mobilen Applikationen bis hin zu digitalen Nachschlagewerken auf breiter Front Einzug gehalten. Trotzdem reicht ein alltäglicher Umgang mit dem Smartphone nicht aus, um die digitalen Kompetenzen zu erlangen, die für einen sicheren und sinnvollen Einsatz im medizinischen Alltag nötig sind [13]. Eine professionelle Ausbildung in digitaler Medizin, welche ein breites Portfolio von Kompetenzen von rechtlichen Aspekten bis hin zum Umgang mit virtuel-

\section{Kontrollierte Studien, welche die Sicherheit und Wirksamkeit solcher Programme nachweisen könnten, sind dringend nötig.}

len Patientenkontakten und Smart Devises umfasst, muss ein obligatorischer Teil der Aus- und Weiterbildung aller Ärzte werden [14].

Es wäre ein leichtfertiges Vergeben der Chancen, welche die digitale Notfallmedizin zur Bewältigung der oben beschriebenen Probleme bietet, wenn digitale Kompetenzen sowohl bei Ärzten als auch bei Patienten nicht strukturiert gefördert werden würden [15].

\section{Ausblick und Zukunftsmusik}

Wenn wir die Chancen, die die Digitalisierung für die Notfallversorgung bietet, ergreifen wollen, ist eine Akademisierung der eNotfallmedizin mit einer wis- 
senschaftlichen Begleitung von telenotfallmedizinischen Projekten von grosser Wichtigkeit und darf nicht ausschliesslich der Industrie überlassen werden. Idealerweise wird diese Zukunft von allen in der Akutmedizin und Notfallversorgung tätigen Ärzten in interprofessioneller Zusammenarbeit mit allen Gesundheitsberufen mitgestaltet. Einen ersten Beitrag hierzu wollen wir am Universitären Notfallzentrum des Insel-

\section{Die Ausbildung in digitalen Kompetenzen muss ein fester Teil jedes medizinischen Curriculums werden}

spitals mit der neu etablierten Abteilung für eNotfallmedizin leisten. Ebenfalls wird dieser Abteilung ab 2020 eine Stiftungsprofessur für Telenotfallmedizin angegliedert sein [16].

Die Ausbildung in digitalen Kompetenzen muss ein fester Teil jedes medizinischen Curriculums werden und auf allen Stufen der medizinischen Ausbildung vom Medizinstudium bis hin zur ärztlichen Weiterbildung implementiert sein.

Mit diesen Voraussetzungen sind wir überzeugt, dass die eNotfallmedizin eine grosse Chance für die Notfallversorgung der Zukunft ist und dazu beitragen kann,

\section{Das Wichtigste in Kürze}

- Wie auch im ambulanten Bereich nimmt die Zahl der Notfallkonsultationen in an Krankenhäuser angeschlossenen Notfallstationen stetig zu.

- Eine telemedizinische, videounterstützte Beratung in Echtzeit wird aktuell von weniger als $10 \%$ der Ärzte in der Schweiz angeboten.

- Um die eNotfallmedizin akademisieren zu können, etabliert das Universitäre Notfallzentrum des Inselspitals eine Abteilung für eNotfallmedizin mit angegliederter Stiftungsprofessur fürTelenotfallmedizin.

- Eine professionelle Ausbildung in digitaler Medizin muss ein obligatorischer Teil der Aus- und Weiterbildung aller Ärzte werden.

\section{L'essentiel en bref}

- Comme dans le secteur ambulatoire, le nombre de consultations en urgence dans les services d'urgence rattachés aux hôpitaux ne cesse d'augmenter.

- Actuellement, la consultation télémédicale par vidéo, en temps réel, est proposée par moins de $10 \%$ des médecins en Suisse.

- Afin de pouvoir enseigner la télémédecine d'urgence, le Centre universitaire des urgences de I'Inselspital ouvre un service de télémédecine d'urgence, avec une chaire dédiée qui lui sera rattachée. die Versorgung der Notfallpatienten auf hohem Niveau zu halten und Herausforderungen wie den Ärztemangel in der Notfall- und Hausarztmedizin konstruktiv anzugehen und abzufedern.

\section{Dank}

Das Universitäre Notfallzentrum dankt dem Touring Club Schweiz für die Unterstützung der eNotfallmedizin-Forschung am Universitären Notfallzentrum durch die Stiftungsprofessur Telenotfallmedizin am Universitären Notfallzentrum.

\section{Literatur}

1 Stücheli B. Als Hausarzt leiste ich Notfalldienst, zumindest im Moment noch. Schweiz Ärzteztg [Internet]. 2019 [cited 2019 Aug 16] Available from: https://doi.emh.ch/saez.2019.18005

2 Senn N, Ebert S, Cohidon C. Die Hausarztmedizin in der Schweiz - Perspektiven. Analyse basierend auf den Indikatoren des Programms SPAM (Swiss Primary Care Active Monitoring) [Internet]. [Cited 2019 Aug 16]. Available from: https://www.obsan. admin.ch/sites/default/files/publications/2016/obsan bulletin 2016-11 d.pdf

3 Ummenhofer W, Siebenpfund P, Stücheli B. Notfall werden ist nicht schwer, Notfall sein dagegen sehr. Schweiz Ärzteztg [Internet]. 2019 [cited 2019 Aug 16]. Available from: https://doi.emh. ch/saez.2019.18006

4 Exadaktylos A, Hautz W. Emergency Medicine in Switzerland. ICU Manag Pract [Internet]. 2015;15. Available from: https:// healthmanagement.org/c/icu/issuearticle/emergency-medicinein-switzerland

5 Bertelsmann Stiftung (Hrsg.), editor. \#SmartHealthSystems: Auszug Schweiz Digitalisierungsstrategien im internationalen Vergleich [Internet]. [Cited 2019 Aug 16]. Available from: https:// www.bertelsmann-stiftung.de/de/publikationen/publikation/did/ smarthealthsystems-auszug-schweiz/

6 Golder L, Jans C. Swiss eHealth Barometer 2019: Schlussbericht Gesundheitsfachpersonen [Internet]. gfs.bern, editor. Available from: https://www.gfsbern.ch/DesktopModules/EasyDNNNews/ DocumentDownload.ashx?portalid=0\&moduleid=677\&articleid $=$ 1585\&documentid=1511

7 Gann B. Giving patients choice and control: health informatics on the patient journey. Yearb Med Inform. 2012;7:70-3.

8 Rat C, Hild S, Rault Sérandour J, Gaultier A, Quereux G, Dreno B, et al. Use of Smartphones for Early Detection of Melanoma: Systematic Review. J Med Internet Res. 2018;20:e135.

9 Semigran HL, Linder JA, Gidengil C, Mehrotra A. Evaluation of symptom checkers for self diagnosis and triage: audit study. BMJ. 2015; h3480.

10 Fraser H, Coiera E, Wong D. Safety of patient-facing digital symptom checkers. The Lancet. 2018;392:2263-4.

11 Patterson BW, Pulia MS, Ravi S, Hoonakker PLT, Schoofs Hundt A, Wiegmann D, et al. Scope and Influence of Electronic Health Record-Integrated Clinical Decision Support in the Emergency Department: A Systematic Review. Ann Emerg Med. 2019;74:285-

12 Attia ZI, Noseworthy PA, Lopez-Jimenez F, Asirvatham SJ, Deshmukh AJ, Gersh BJ, et al. An artificial intelligence-enabled ECC algorithm for the identification of patients with atrial fibrillation during sinus rhythm: a retrospective analysis of outcome prediction. The Lancet [Internet]. 2019 [cited 2019 Aug 16]. Available from: https://linkinghub.elsevier.com/retrieve/pii/ S0140673619317210

13 Kuhn S. Medizin im digitalen Zeitalter: Transformation durch Bildung. Dtsch Arztebl Int [Internet]. 2018. Available from https://www.aerzteblatt.de/int/article.asp?id=197293

14 Gilli Y. Mobile Health holt uns ein - mit welchen Konsequenzen? Schweiz Ärzteztg. 2017;98:359.

15 Zingg T, Sojer R, Röthlisberger F. Digitalisierung in der ambulanten Gesundheitsversorgung. Schweiz Ärzteztg [Internet]. 2019 [cited 2019 Jul 31]. Available from: https://doi.emh.ch/saez.2019.17521

16 Pressemitteilung www.unibe.ch, Neue Stiftungsprofessur für Telenotfall medizin 